\title{
KINEMATIC SYNTHESIS OF CENTRAL-LEVER STEERING MECHANISM FOR FOUR WHEEL VEHICLES
}

\author{
Santiranjan PramaniK ${ }^{a, b, *}$, Sukrut Shrikant Thipse ${ }^{c}$ \\ ${ }^{a}$ Symbiosis International (Deemed University), Faculty of Engineering, Department of Mechanical Engineering, \\ Lavale, Mulshi, Pune, Maharashtra 412115, India \\ ${ }^{b}$ College of Military Engineering, Department of Mechanical Engineering, Old Mumbai - Pune Hwy, Dapodi, \\ Pimpri-Chinchwad, Maharashtra 411031, India \\ ${ }^{c}$ The Automotive Research Association of India, Survey No.102, Paud Road, Rambaug Colony, Kothrud, Pune, \\ Maharashtra 411038, India \\ * corresponding author: santiranjan_pramanik@rediffmail.com
}

\begin{abstract}
A central lever steering mechanism has been synthesized to obtain five precision points for a four-wheel vehicle using Hooke and Jeeves optimization method. This compound mechanism has been studied as two identical crossed four-bar mechanisms arranged in series. The optimization has been carried out for one crossed four-bar mechanism only instead of the entire mechanism. The number of design parameters considered for the optimization is two. The inner wheel has been considered to rotate up to 52 degrees. The steering error, pressure angle and mechanical advantage of the proposed mechanism have been compared with those achieved by the Ackermann steering mechanism. The proposed mechanism has less steering error, more favourable pressure angle and increased mechanical advantage. The method of compounding the mechanism is also applicable when the central lever is offset from the longitudinal axis of the vehicle.
\end{abstract}

KEYwords: Ackermann steering, four-wheel vehicle, Hooke and Jeeves method, compound steering mechanism, unsymmetrical mechanism, steering error, crossed four-bar mechanism.

\section{INTRODUCTION}

There are several types of steering mechanisms used for four-wheel vehicles. There are symmetric fourbar mechanism, centre-lever mechanism and rack-andpinion type mechanism. Fahey and Huston [1] considered an eight-bar mechanism for steering automobiles. They suggested this mechanism after modifying a leading Ackermann steering mechanism. This eight-bar mechanism eliminated the divergent end behaviour [1] of the existing Ackermann mechanism and the maximum steering error was reduced to 0.03 degree. The progressive deviation in the steering error curve with the increasing rotation of the inner wheel nearing the end range is referred here as divergent-end behaviour. However, there were two small and disproportionate links in the eight-bar mechanism and hence the mechanism was unpractical.

Simionescu and Smith [2] found out initial estimates for the design of centre-lever steering linkages. They considered both leading and trailing mechanisms for which they produced parametric design charts.

De-Juan et al. 3 considered kinematic synthesis of six-bar steering linkages for both leading and trailing configurations. They considered mixed-leading and mixed-trailing configurations as well. In their work, it was found that the steering error curve had five precision points for leading configuration only.

De-Juan et al. 4 considered three types of leading steering mechanisms and optimized these considering steering error and transmission angles and compared them.

Bajpai et al. [5] explained optimization of a function of several variables by Hooke and Jeeves method, which was a direct method in which it was not necessary to differentiate the function with respect to the variables.

Zhao et al. [6] proposed a geared five-bar steering mechanism with noncircular gear sectors capable to exactly satisfy Ackermann's formula. However, manufacture imperfections and backlash can produce steering error. They suggested that this mechanism can be applied to light carriages.

Peterson and Kornecki [7] considered a design of the steering mechanism of a wide power frame used to harvest tree fruits. The unit was collapsible to half the width for the purpose of transportation. The ratio of the wheel track to wheelbase was 1.55 while harvesting fruits and 0.77 while being transported on the road.

A crossed four-bar steering mechanism that included two spur gears has been considered in reference 8 . There, Hooke and Jeeves method has been used to minimize the objective function that comprised of steering error only. The present mechanism has been considered to consist of two such crossed four-bar mechanisms to eliminate the spur gears. The mechanism provides five precision points and is a trailing mechanism. The kinematic synthesis has been necessary for crossed four-bar mechanism only and 
there are two design parameters, thus simplifying the calculation.

The aim of the present work is to carry out kinematic synthesis of a centre lever steering mechanism in a simplified approach. The kinematic synthesis of the offset centre lever steering mechanism has been considered here for the first time. It was found that the offset centre lever steering mechanisms can be designed easily if the central hinge is not imposed to be located on the longitudinal axis of the vehicle.

\section{Crossed Four-BAR STEERING MECHANISM}

Figure 1 shows a crossed four-bar steering mechanism. The vehicle has been turned towards right. $A G F B$ is the crossed four-bar mechanism. The inner wheel is rotated about centre I and the outer wheel has been rotated about centre $A$. When the inner wheel rotates by angle $F B E$, then the outer wheel rotates by angle $G A D$. As the steering arm $B F$ is rotated counter clockwise, the steering arm $A G$ is rotated clockwise. Since these are rotating in opposite directions the use of spur gears has become necessary.

\section{Condition of CORRECT STEering}

The steering is assumed geometrically correct if the axes of rotation of the wheels intersect at the instantaneous centre of rotation of the vehicle relative to the ground. Figure 2 shows that when the inner wheel rotates by an angle $\gamma$, then the outer wheel rotates by an angle $\alpha$. The steering position is correct as all four wheels rotate about a common centre $O$. From this geometry the following equation can be written:

$$
\cot \alpha-\cot \gamma=\frac{t}{w}
$$

\section{Optimization method}

The mechanism shown in Figure 3 has two parameters. These are angle $K A G(\beta)$ and steering arm length $A G(r)$. The inner wheel has been rotated by angle $\phi$ (angle $F B E$ ) and then the rotation of the outer wheel (angle $G A D$ ) has been found out. The correct rotation of the outer wheel has been found out using an equation (1). Then the steering error has been calculated as follows.

The initial straight-ahead position has two equal angles $K A G$ and $F B H$. The two steering arms $A G$ and $F B$ are equal. The length of the coupler has been found as

$$
\begin{gathered}
G F=\sqrt{(d-2 r \sin \beta)^{2}+(2 r \cos \beta)^{2}} \\
\text { Angle } B A E=\tan ^{-1}\left(\frac{B E V}{A E H}\right)
\end{gathered}
$$

where

$$
B E V=r \sin \left(\frac{\pi}{2}-\beta+\phi\right)
$$

and

$$
A E H=d-r \cos \left(\frac{\pi}{2}-\beta+\phi\right)
$$

$$
\text { Angle } D A E=\cos ^{-1}\left\{\frac{r^{2}+(A E)^{2}-(G F)^{2}}{2 \times r \times A E}\right\}
$$

where

$$
\begin{aligned}
A E & =\sqrt{(B E V)^{2}+(A E H)^{2}} \\
\text { Angle } D A B & =\text { Angle } D A E-\text { Angle } B A E
\end{aligned}
$$

The rotation of the outer wheel is given by

$$
\text { Angle } G A D=\frac{\pi}{2}-\beta-\text { Angle } D A B
$$

The correct angle of rotation of the outer wheel is given by

$$
\text { Angle } G A D_{\text {correct }}=\cot ^{-1}\left(\cot \phi+\frac{t}{w}\right)
$$

The steering error is given by

$$
\text { Error }=\text { Angle } G A D-\text { Angle } G A D_{\text {correct }}
$$

The objective function is given by

$$
\text { Obj fun }=\sum \text { Error }^{2}
$$

The limits of the above sum are $\phi$ from zero to 52 degree. The design variables are $\beta$ and $r$.

Hooke and Jeeves optimization method [5] has been applied to minimize the objective function $(12)$. We use, as initial estimate, $r=1.9374$ and angle $\beta=13.5407^{\circ}$ obtained by an analytical method in another study [9]. A vehicle with a track to wheelbase ratio 0.326 has been considered, for which the final estimate has been made. Using the concept of compound mechanisms, the track to wheelbase ratio has been taken as 0.163. By optimization, we found the length of the steering arm 1.933 units and inclination angle $\beta$ as 13.521 degree, where the distance $A B$ is 10 units. The steering arm angles are $\beta=13.521$ degree. We have shown the steering error curve in Figure 4 The maximum steering error of the proposed compound mechanism has been reduced to 0.044 degree by the through optimization. The steering error of the proposed mechanism is less compared to that of the Ackermann steering mechanism of the benchmark vehicle [10].

The pressure angle at any joint is defined as the angle between the output link and the direction of the force applied by the input link. The pressure angles at joint $D$ and $E$ are found out as follows:

$$
\text { Angle }(A D E)=\cos ^{-1}\left\{\frac{r^{2}+(G F)^{2}-(A E)^{2}}{2 \times r \times G F}\right\}
$$

The pressure angle at joint $D$ is given by

$$
\mu_{1}=\left\{\text { Angle }(A D E)-\frac{\pi}{2}\right\}
$$




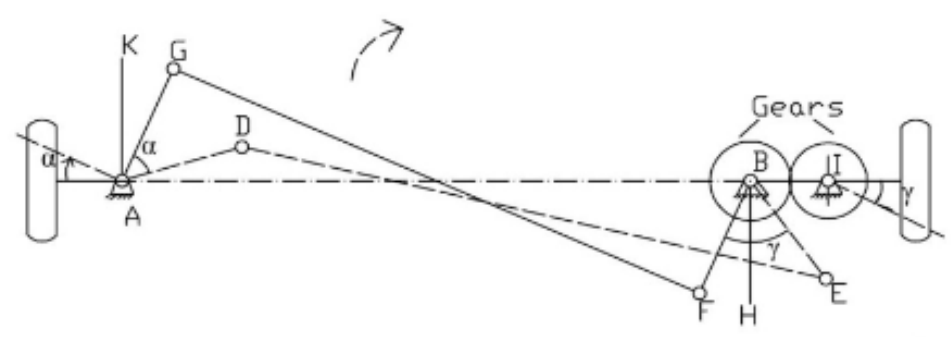

FiguRE 1. Crossed four-bar steering mechanism including gears.

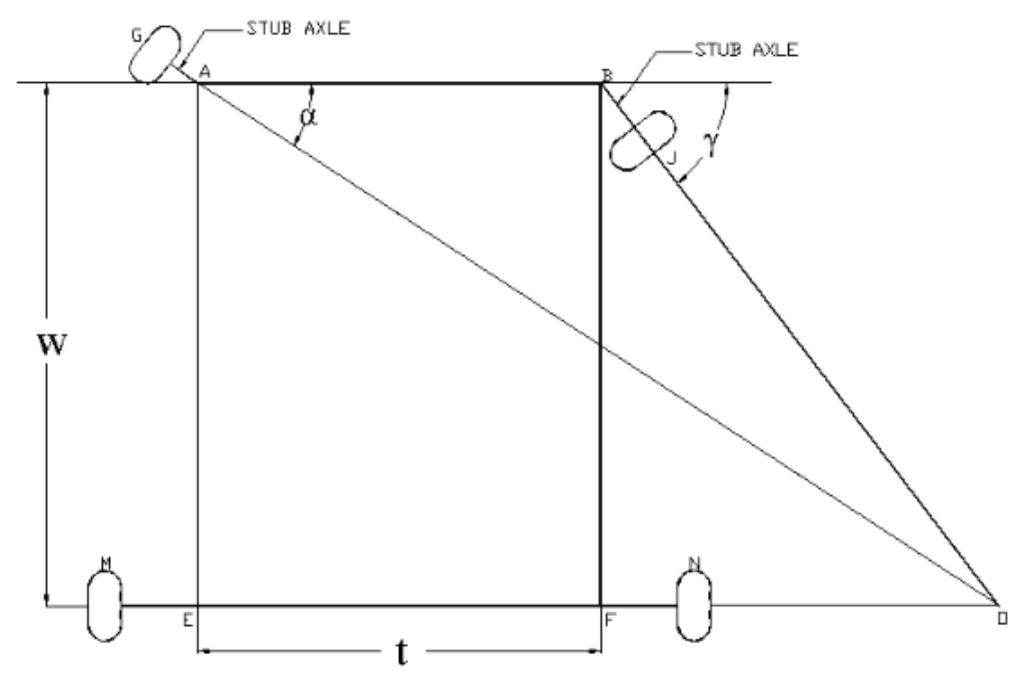

FigURE 2. Geometry of correct steering.

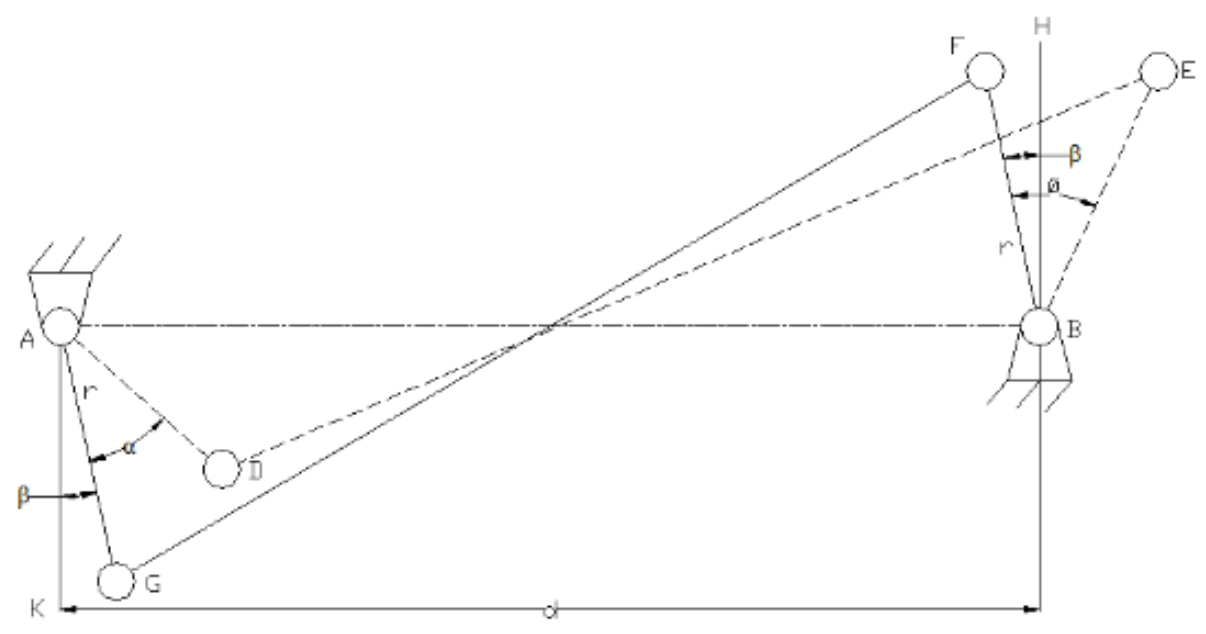

Figure 3. Geometry of a crossed four bar mechanism. 


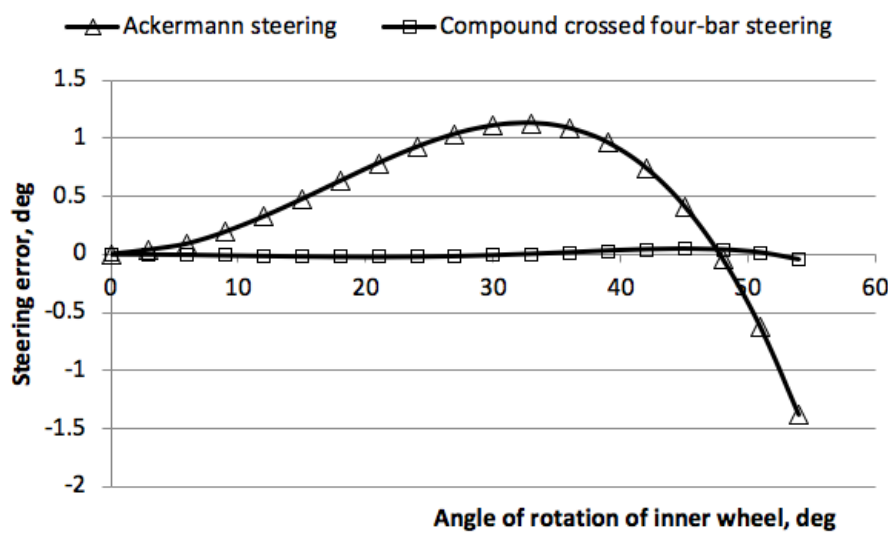

FiguRE 4. Comparison of steering error.

Now the length $D B$ is given by

$$
D B=\sqrt{\{d-r \cdot \cos (D A B)\}^{2}+\{r \cdot \sin (D A B)\}^{2}}
$$

The angle $(B E D)$ is given by

$$
\text { Angle }(B E D)=\cos ^{-1}\left\{\frac{r^{2}+(D E)^{2}-(D B)^{2}}{2 \times r \times D E}\right\}
$$

The pressure angle at joint $E$ is given by

$$
\mu_{2}=\left\{\frac{\pi}{2}-\operatorname{Angle}(B E D)\right\} .
$$

The pressure angle of the proposed steering mechanism has been compared with that of the conventional Ackermann steering of the benchmark vehicle [10]. As Figure 5 shows, the pressure angle of the proposed compound steering mechanism is less than that of the Ackermann steering mechanism.

Mechanical advantage of a mechanism is defined as the ratio of the torque produced in the output link and the torque provided in the input link. This has been calculated [9] from the product $\left(\cos \mu_{1} \times \cos \mu_{2}\right)$.

The mechanical advantages of the two mechanisms have been compared in the Figure 6 and it has been found that the proposed mechanism has a higher mechanical advantage.

\section{COMPOUnd SIX MEMBER MECHANISM}

A compound six-member mechanism has then been considered, comprising of two mirrored crossed fourbar mechanisms. The compound mechanism has a track to wheelbase ratio 0.326 , which is twice the ratio of 0.163 . The track to wheelbase ratio 0.163 for a vehicle is not feasible, but using ratio 0.163 after compounding effect is suitable as the vehicle has the said ratio 0.326 which is used in benchmark vehicle12 M, a bus by Ashok Leyland [10] which is the same as above. The front wheel track of this vehicle is $2020 \mathrm{~mm}$ which is shown as 20 units in Figure 7. Hence one unit is $101 \mathrm{~mm}$ in this figure. The wheelbase of the vehicle is $6200 \mathrm{~mm}$. The length of the arms $C E, B D, B F$ and $A G$ is 1.933 units, which are equal to $195.2 \mathrm{~mm}$. The length of the coupler $G F$ and $D E$ is $994 \mathrm{~mm}$.

The crossed four bar mechanism is such that two such chains can be series connected as shown in the Figure 7. Here, the central lever BFD can be moved backwards in order to increase the space for installing the engine and the gearbox. The paper under reference [8] uses spur gears to bring the mechanism behind the front axle and the use of gears may produce more steering error. So the mechanism suggested in the present paper has two benefits. The first benefit is the availability of more space and the second one is the elimination of the spur gears. The Figure 8 shows the modification of the mechanism to increase the available space. The present paper shows the usefulness of crossed four bar chain. In this figure, the central hinge joint is shifted backwards by the distance $O G$, which is $520 \mathrm{~mm}$, for example. Now the distance $G F$ and $A G$ is $1136 \mathrm{~mm}$. The arms $A B, G C, G D$ and $E F$ are all $219.6 \mathrm{~mm}$ in length. The length of the coupler $D E$ and $B C$ is $1118 \mathrm{~mm}$ each. The length of the link $C D$ is $286.8 \mathrm{~mm}$.

In case there is such a constraint that the hinge point $G$ cannot be placed on the longitudinal axis of the vehicle, then this can be accommodated by modifying the mechanism as shown in the Figure 9 The kinematic synthesis for such an offset centre lever mechanism is difficult by the presently available methods. But the concept of compounding the mechanism is very useful to devise an offset centre lever steering mechanism.

In Figure 9 it has been shown that the central hinge $G$ has been located by distances $x$ and $y$. This shift is not done intentionally but is required when there is a constraint that the central hinge cannot be placed on the longitudinal axis. Here, the distances $x$ and $y$ have been chosen arbitrarily as an example. The links $A B$ and $G C$ are equal in size. Also, the links $E F$ and $G D$ are equal in length. For a non-zero value of the distance $x$, the links $G C$ and $G D$ are unequal. In this 


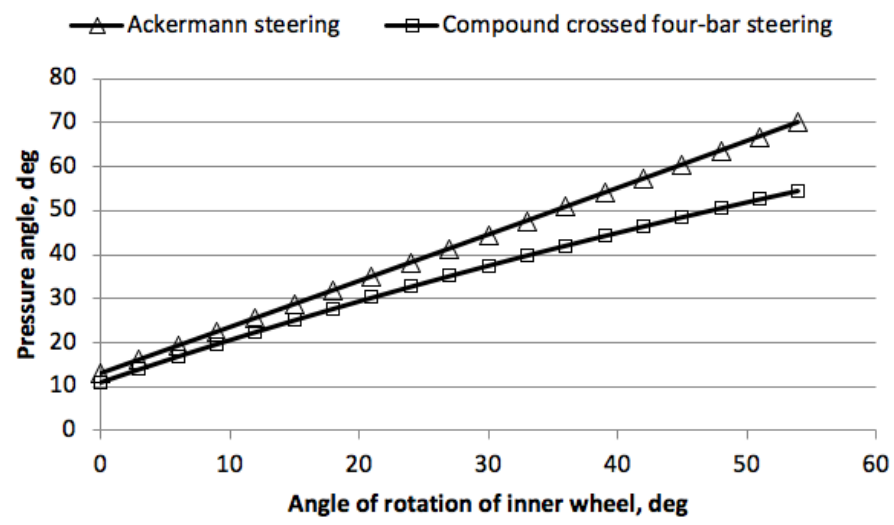

Figure 5. Comparison of pressure angles.

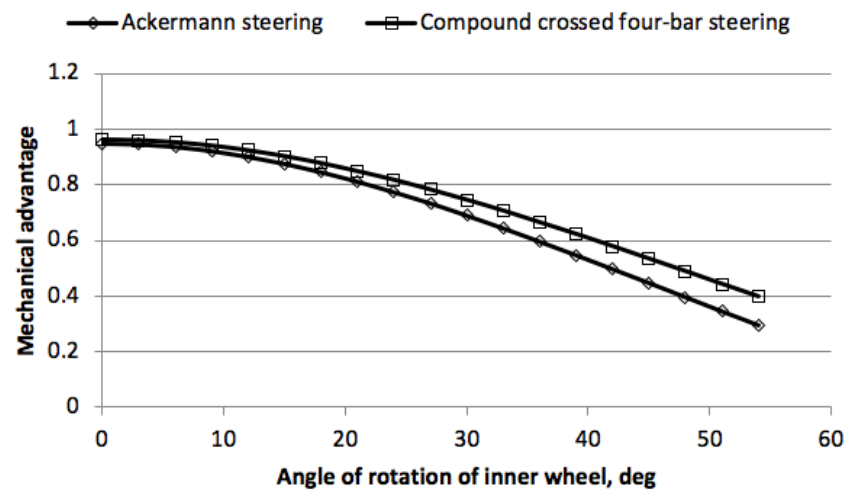

Figure 6. Comparison of mechanical advantages.

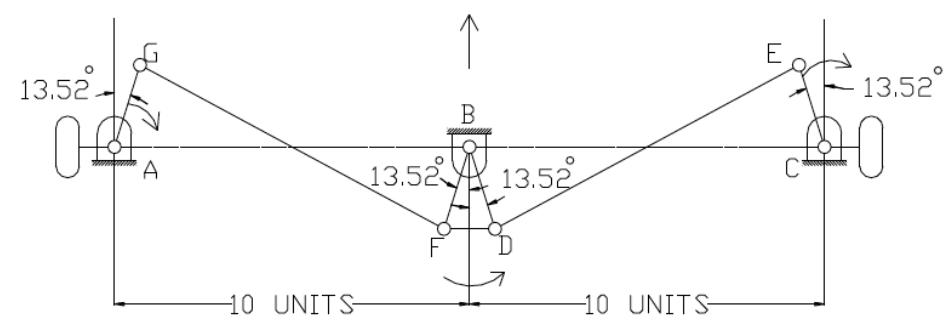

Figure 7. Compound six bar mechanism. 


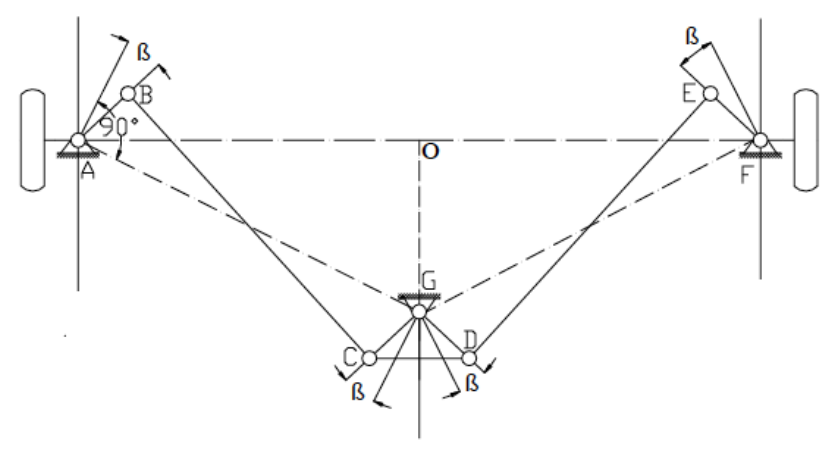

FIgURE 8. Modification of the mechanism to increase space.

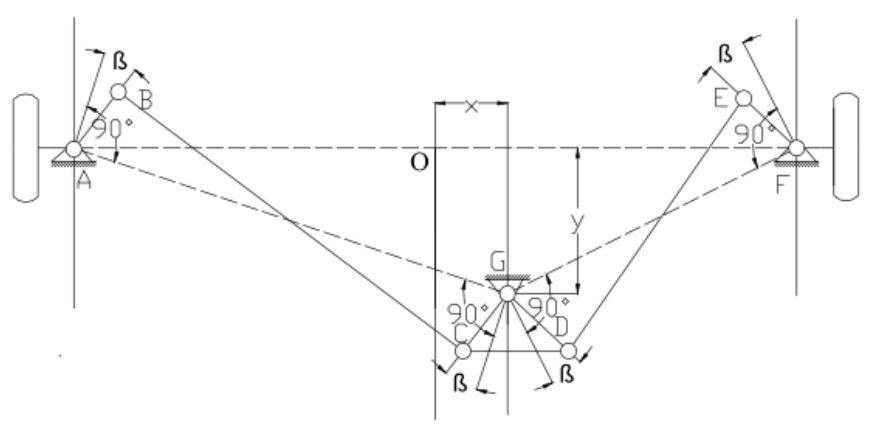

Figure 9. Offset center lever steering mechanism.

figure, for example, $x$ is $200 \mathrm{~mm}$ and $y$ is $500 \mathrm{~mm}$. The distance $A O$ and $F O$ are equal to half of the front track, which is $2020 \mathrm{~mm}$. Using Pythagorean theorem, we find the distances $A G$ and $G F$ as $1309.2 \mathrm{~mm}$ and $951.9 \mathrm{~mm}$ respectively. The arms $E F$ and $G D$ are $184 \mathrm{~mm}$ in length. But the arms $A B$ and $G C$ each are $253.1 \mathrm{~mm}$ in length. The length of the coupler $B C$ is $A G \times 994 / 1010=1288.5 \mathrm{~mm}$. The length of the coupler $D E$ is $G F \times 994 / 1010=936.8 \mathrm{~mm}$. The length of the link $C D$ is calculated as follows.

The angle $C G D$ is given by

$$
\begin{gathered}
\tan ^{-1}\left(\frac{Y}{O A+X}\right)+\tan ^{-1}\left(\frac{Y}{O F-X}\right)+2 \beta= \\
\tan ^{-1}\left(\frac{500}{1010+200}\right)+\tan ^{-1}\left(\frac{500}{1010-200}\right)+ \\
+2 \times 13.521= \\
22.452+31.686+27.042=81.18^{\circ}
\end{gathered}
$$

From the triangle $C G D$ the following equation can be written

$$
\begin{array}{r}
C D^{2}=G C^{2}+G D^{2}-2 \times G C \times G D \times \cos (C G D)= \\
=184^{2}+253.1^{2}-2 \times 184 \times 253.1 \times \cos \left(81.18^{\circ}\right)= \\
83634.26
\end{array}
$$

Therefore, the length of the link $C D$ is $289.2 \mathrm{~mm}$.
The steering error curve for mechanisms shown throughout Figure 7 to Figure 9 should be same as all of these are obtained by compounding two crossed four-bar mechanisms having similar proportions. The steering error curve has been shown in Figure 10 which shows that by compounding effect, the steering error has been reduced from that of a single crossed four bar mechanism that employs two equal spur or helical gears.

\section{Conclusions}

It has been shown that two crossed four-bar chains can be added in series to obtain a centre lever steering mechanism. The kinematic synthesis of this centre lever steering mechanism has been found to be easy in the present work. The mechanism can be suitably modified to provide more space for the placement of the engine, gearbox and other parts of the vehicle. This mechanism is more accurate than the conventional Ackermann steering mechanism and also the crossed four bar steering mechanism. This mechanism has a low pressure angle and high mechanical advantage compared to the Ackermann steering mechanism.

Also, it can accommodate a constraint like the requirement of the placement of the central hinge at some offset from the longitudinal axis of the vehicle. In this case, two crossed four-bar chains are not iden- 


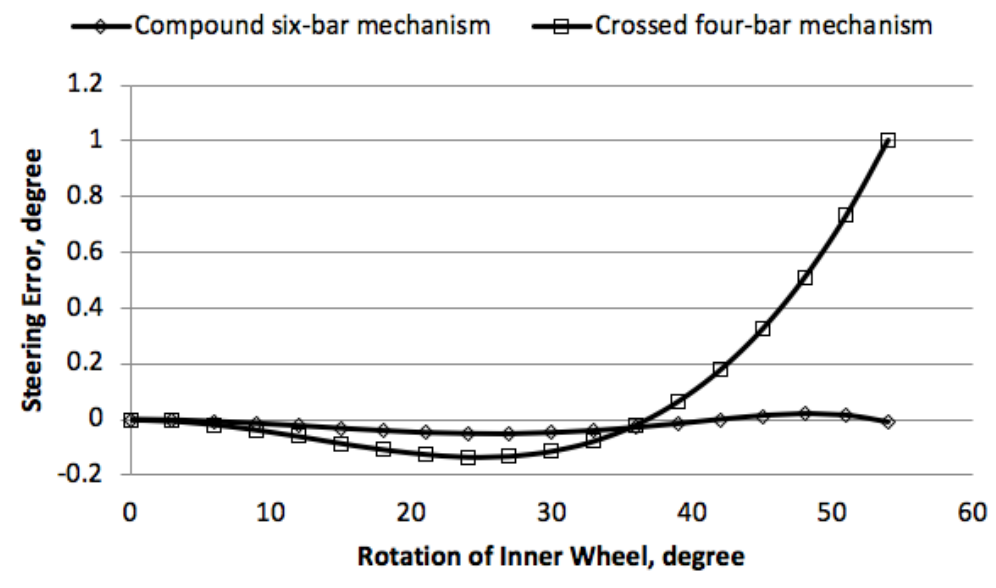

Figure 10. Comparison of steering error.

tical as one is smaller in size and the other is bigger in size because of the offset. The steering arms are unequal in length. But both have been designed for equal value of the wheel track by a wheel base ratio 0.163 .

In the case of a wide track vehicle mentioned in reference [7], the steering mechanism has to be made by adding four numbers of crossed four-bar chains each having an imaginary track to wheelbase one-fourth of that of the expanded vehicle while harvesting fruits. While this vehicle has to be transported on the road, it can be made collapsible by using two crossed fourbar chains in series by discarding two such four-bar chains.

\section{REFERENCES}

[1] S. O. Fahey, D. R. Huston. A Novel Automotive Steering Linkage. Journal of Mechanical Design 119(4):481 - 484, 1997. DOI:10.1115/1.2826393

[2] P. A. Simionescu, M. R. Smith. Initial Estimates in the Design of Central-Lever Steering Linkages . Journal of Mechanical Design 124(4):646 - 651, 2002. DOI:10.1115/1.1505853

[3] A. De-Juan, R. Sancibrian, F. Viadero. Optimal synthesis of function generation in steering linkages. International Journal of Automotive Technology 13(7):1033 - 1046, 2012. DOI:10.1007/s12239-012-0106-4
[4] A. De-Juan, R. Sancibrian, F. Viadero. Optimal synthesis of steering mechanism including transmission angles. In Proceedings of EUCOMES, pp. 177 - 183. 2008.

[5] A. C. Bajpai, L. R. Mustoe, D. Walker. Advanced Engineering Mathematics. John Wiley and Sons, London, 1990.

[6] J. S. Zhao, X. Liu, Z. J. Feng, J. S. Dai. Design of an Ackermann type steering mechanism. Journal of Mechanical Engineering Science 227(11):2549 - 2562, 2013. DOI:10.1177/0954406213475980

[7] D. L. Peterson, T. S. Kornecki. Steering mechanism for wide-track vehicles. Applied Engineering in Agriculture 2(1):16 - 17, 1986. DOI:10.13031/2013.26699

[8] S. Pramanik, S. S. Thipse. Kinematic synthesis of a crossed four-bar mechanism for automotive steering. International Journal of Vehicle Structures and Systems 9(3):169 - 171, 2017. DOI:10.4273/ijvss.9.3.07.

[9] S. Pramanik. Design and Development of Innovative Crossed Four-bar Steering Mechanism for Automobiles. Ph.D. thesis, Symbiosis International University, Pune, India, 2019.

[10] Wikipedia. Ashok Leyland 12M. https://en.wikipedia.org/wiki/Ashok_Leyland_12M 2019. Accessed: April 2020. 